\title{
Inhibition of antiviral drug cidofovir on proliferation of human papillomavirus-infected cervical cancer cells
}

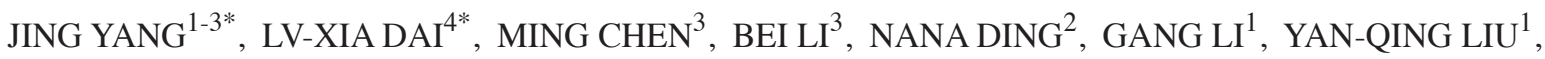 \\ MING-YUAN LI ${ }^{2}$, BAO-NING WANG ${ }^{2}$, XIN-LI SHI $^{5}$ and HUA-BING TAN ${ }^{1}$
}

\author{
${ }^{1}$ Department of Infectious Disease, Renmin Hospital, Hubei University of Medicine, Shiyan, Hubei 442000; \\ ${ }^{2}$ Department of Microbiology, West China School of Preclinical and Forensic Medicine, Sichuan University, Chengdu, \\ Sichuan 610041; ${ }^{3}$ Department of Microbiology, School of Basic Medical Sciences, Hubei University of Medicine, Shiyan, \\ Hubei 442000; ${ }^{4}$ Experiment Teaching Center of Clinical Medicine, Chengdu College of Medicine, Chengdu, Sichuan 610500; \\ ${ }^{5}$ Department of Pathobiology and Immunology, Hebei University of Chinese Medicine, \\ Shijiazhuang, Hebei 050200, P.R. China
}

Received April 22, 2015; Accepted July 22, 2016

DOI: $10.3892 / \mathrm{etm} .2016 .3718$

\begin{abstract}
In order to evaluate the potential application value of cidofovir (CDV) in the prevention of human papillomavirus (HPV) infection and treatment of cervical cancer, the inhibitory effect of CDV on the proliferation of HPV 18-positive HeLa cells in cervical cancer was preliminarily investigated, using cisplatin (DDP) as a positive control. An MTT assay was used to analyze the effects of CDV and DDP on HeLa cell proliferation. In addition, clone formation assay and Giemsa staining were used to examine the extent of HeLa cell apoptosis caused by CDV and DDP. Flow cytometry was also used to detect the shape and size of apoptotic cells following propidium iodide staining, while western blot analysis identified the expression levels of of E6 and p53 proteins in HeLa cells. A cell climbing immunofluorescence technique was used to locate the subcellular position of p53 in HeLa cells. The results demonstrated that CDV and DDP inhibited the proliferation of HeLa cells in a concentration- and time-dependent manner. Flow cytometry showed that CDV and DDP treatments resulted
\end{abstract}

Correspondence to: Dr Xin-Li Shi, Department of Pathobiology and Immunology, Hebei University of Chinese Medicine, 3 Xingyuan Road, Shijiazhuang, Hebei 050200, P.R. China

E-mail: shixinli_doc@163.com

Dr Hua-Bing Tan, Department of Infectious Disease, Renmin Hospital, Hubei University of Medicine, 30 South Renmin Road, Shiyan, Hubei 442000, P.R. China

E-mail: renmthb@163.com

${ }^{*}$ Contributed equally

Abbreviations: HPV, human papillomavirus; CDV, cidofovir; DDP, cisplatin; PBS, phosphate buffer; E6-AP, E6-associated protein

Key words: cervical cancer, human papillomavirus, HeLa cells, cidofovir, cisplatin, apoptosis in cell arrest in the S-phase, and triggered programmed cell death. Furthermore, western blot analysis revealed that CDV and DDP inhibited E6 protein expression and activated p53 expression in HeLa cells. Finally, the immunofluorescence results indicated that CDV and DDP inhibited the nuclear export of p53 by E6 protein, which is required for degradation of endogenous p53 by MDM2 and human papilloma virus E6. In conclusion, CDV and DDP inhibited HeLa cell proliferation in a concentration- and time-dependent manner, reduced the expression of E6 protein, and reinstated p53 protein activity. Thus, CDV regulates cell cycle arrest and apoptosis, and may be a potential cervical cancer therapeutic strategy.

\section{Introduction}

Cervical cancer ranks as the second most common cause of mortality in women with malignant tumors. Each year, $\sim 450,000$ women are newly diagnosed with cervical cancer worldwide, with $80 \%$ of cases occurring in developing countries, and with mortality observed in $\sim 280,000$ of these cases (1). In China, $\sim 130,000$ new cases of cervical cancer are annually diagnosed, accounting for $28 \%$ of the total cases diagnosed worldwide (2-4). Currently, cervical cancer is the only malignant tumor that can be definitively diagnosed and possibly prevented. Human papillomavirus (HPV) serves a key role in cancer development processes, and the persistent infection of HPV is considered to be the most important cause of cervical cancer development. In total, $80 \%$ of women will be infected with HPV during their lifetime; however, in the majority of cases the body is able to clear the virus within 24 months without treatment (5). Therefore, only a small number of women are infected persistently, which can result in HPV infection progressing into precancerous lesions and eventually invasive cervical cancer after $10-15$ years (6). To date, $>180$ subtypes of HPV have been identified, including HPV 16 and HPV 18, which are two high risk HPV subtypes and the main causes of cervical cancer due to persistent infection of host cells. E6 and E7 are two genes expressed in 
these high risk HPVs, and are the main oncogenes of cervical cancer (7).

To date, HPV treatment focuses on the symptoms of the infection. If the HPV infection causes abnormal cell changes, there are four main treatment options: Cryotherapy, surgical removal, laser therapy and loop electrosurgical excision procedure. Primary prevention with HPV vaccination remains the most effective strategy; however, vaccines may not offer protection against all cancer-associated HPV types. Furthermore, there is still no FDA approved anti-HPV drug listed so far but the search for potential drug candidates against HPV strains is increasing. The current anti-HPV drugs are predominantly oral hormonal medicines, including acyclovir, ganciclovir, interferon and interleukin. Nevertheless, a number of traditional Chinese medicines (including Chaihu, Youdujing, Paiteling and Xinfuning) have been used for the prevention and treatment of HPV.

In terms of treatment, cidofovir that is formulated as a gel can be safe and effective for the treatment of epithelial hyperplasia in those with human immunodeficiency virus infection (8). Furthermore, chemotherapy is considered as the standard treatment for patients with advanced or recurrent cervical cancer, and cisplatin appears to treat the disease effectively.

The present study aimed to compare the inhibitory effects of cidofovir (CDV) on the proliferation of HPV-18 positive HeLa cells with the effect of cisplatin (DDP), in order to evaluate the potential application value of CDV in the prevention and treatment of cervical HPV infection.

\section{Materials and methods}

Reagents. Dulbecco's modified Eagle's medium (DMEM) and penicillin-streptomycin solution used in cell cultures were purchased from Hyclone (GE Healthcare Life Sciences, Logan, UT, USA). Newborn calf serum was purchased from Gibco (Thermo Fisher Scientific, Inc., Waltham, MA, USA). Nuclear and Cytoplasmic Protein Extraction kit (163-2089) was purchased from Bio-Rad Laboratories Inc., (Hercules, CA, USA). Mouse anti-human monoclonal E6 antibody (ab51931) was purchased from Abcam (Cambridge, UK). In addition, anti-mouse FITC secondary antibody (200-032-037) was purchased from Jackson Immunoresearch Inc., (West Grove, PA, USA), while the p53 anti-mouse (BA0521) and anti-mouse $\beta$-actin (BA2305) antibodies were purchased from Boster Biological Technology, Ltd., (Wuhan, China). CDV, Goat anti-mouse IgG-HRP (sc-2005) and anti-rabbit IgG-HRP (sc-2004) were purchased from Santa Cruz Biotechnology Inc., (Shanghai, China). Alexa Fluor 594-conjugated donkey anti-rabbit IgG secondary antibody (R37119) for immunofluorescence staining was purcahsed from Thermo Fisher Scientific, Inc. DDP and dimethyl sulfoxide (DMSO) reagents were purchased from Sigma-Aldrich (St. Louis, MO, USA), all of which were dissolved in $0.01 \mathrm{M}$ phosphate-buffered saline (PBS) to make a $50 \mu \mathrm{M}$ stock solution, and diluted with DMEM to the required concentration prior to use.

HeLa cell culture. HeLa cells (gifted from Professor Liu Cong; West China Second University Hospital) were cultured in complete DMEM supplemented with $10 \%$ fetal bovine serum (both Gibco; Thermo Fisher Scientific, Inc.), $100 \mathrm{U} / \mathrm{ml}$ penicillin and $100 \mu \mathrm{g} / \mathrm{ml}$ streptomycin. Cells were harvested by trypsinization and an assessment was made of their density using a hemocytometer to a density of $1.0 \times 10^{5}$ cells $/ \mathrm{ml}$ and $5.0 \times 10^{4}$ cells were added to each well of a 96-well tissue culture-treated plate (Costar; Sigma-Adrich). The cells were inoculated and then incubated at $37^{\circ} \mathrm{C}$ with $5 \% \mathrm{CO}_{2}$ for 14 days. The DMEM culturing medium was preheated at $37^{\circ} \mathrm{C}$ in a water bath for a minimum of 30-45 mins to ensure they were at the right temperature, and adherent cells were washed with new pre-warmed media and aspirated to remove any traces of the old media. The media was replaced daily. In the present study, the following groups were used: Control group, which included HeLa cells without any drug treatment; CDV-treated group, in which HeLa cells were treated with an appropriate concentration of CDV $(2.5,5,10$ and $20 \mu \mathrm{M})$; and DDP-treated group, in which HeLa cells were treated with an appropriate concentration of DDP $(2.5,5,10$ and $20 \mu \mathrm{M})$.

Detection of HeLa cell viability by MTT assay. HeLa cells in the logarithmic growth phase were selected, digested with $0.25 \%$ trypsin (Gibco; Thermo Fisher Scientific, Inc.), made into cell suspensions of $5 \times 10^{4}$ cells $/ \mathrm{ml}$, and transferred to 96-well plates with $100 \mu \mathrm{l}$ cell suspension in each well. After the cells adhered to the plates, any solution was discarded. Cells were treated with different concentrations of CDV $(2.5,5,10$ and $20 \mu \mathrm{M})$ and DDP $(2.5,5,10$ and $20 \mu \mathrm{M})$ in DMEM for 24, 48 or $72 \mathrm{~h}$. A blank control well was set, and each group was cultured and treated in triplicate. At every time point, $10 \mu 15 \mathrm{mg} / \mathrm{ml}$ MTT solution from an MTT cell proliferation assay kit (11465007001; Sigma-Aldrich) was added into each well and the cells were cultured for a further $4 \mathrm{~h}$. Next, $150 \mu \mathrm{l}$ DMSO was added to terminate the culturing. The plate was then shaken for 10-15 min at a low speed. Subsequently, the optical density (OD) value was identified by enzyme-linked immunosorbent assay using the MTT cell proliferation assay kit (11465007001; Sigma-Aldrich) and a microplate reader (Thermo Fisher Scientific, Inc.) at a wavelength of $490 \mathrm{~nm}$. In addition, the viability of HeLa cells was calculated by the following formula: Cell viability $(\%)=$ $\left(\mathrm{OD}_{490 \mathrm{~nm}}-\mathrm{OD}_{\text {blank }}\right)_{\mathrm{CDV}} /\left(\mathrm{OD}_{490 \mathrm{~nm}}-\mathrm{OD}_{\text {blank }}\right)_{\mathrm{DDP}} \mathrm{X} 100 \%$.

Formation of HeLa cell colonies. HeLa cells in the logarithmic growth phase were selected, digested with $0.25 \%$ trypsin and made into single-cell suspensions with DMEM. Next, the cells were transferred into 6-well plates with $1 \mathrm{ml}$ cell suspension per well, which contained 300 cells per well, and were incubated overnight at $37^{\circ} \mathrm{C}$ with $5 \% \mathrm{CO}_{2}$. Any previous solutions were discarded, and $2.5 \mathrm{ml}$ fresh complete DMEM with corresponding concentrations of drugs were added into each well. The cells were then incubated at $37^{\circ} \mathrm{C}$ with $5 \% \mathrm{CO}_{2}$ for approximately 14 days. A blank control well was set and each group was cultured and treated in triplicate. When visible colonies formed, the culturing was terminated and $2 \mathrm{ml}$ pure methanol was added for $15 \mathrm{~min}$ for cell fixation. Subsequently, any stationary solution was discarded, Giemsa stain (A0909-0010; Applichem $\mathrm{GmbH}$, Darmstadt, Germany) was added for 10-15 min, and then the plates were slowly washed with water and dried in air. Cell colonies containing $>50$ cells were counted under the microscope (low magnification), or by Image-Pro Plus 6.0 software (Media Cybernetics, Inc., Rockville, MD, USA). The 
effects of CDV and DDP on proliferation and apoptosis in HeLa cells were determined based on the survival rate, as follows: Colony formation rate $=$ (number of colonies $/$ number of cells inoculated $) \times 100 \%$; Survival rate $=($ colony formation rate in the drug group / colony formation rate in the control group) $\mathrm{x} 100 \%$. Morphology of the HeLa cells was observed under an inverted microscope (CKX41-A32PH; Olympus Corp., Tokyo, Japan).

Apoptosis determined by Giemsa staining. Sterile coverslips (174950, NUNC, Roskilde, Denmark) were placed into 24-well plates, which were inoculated with $5 \times 10^{5}$ cells per well and incubated overnight at $37^{\circ} \mathrm{C}$ with $5 \% \mathrm{CO}_{2}$. Next, fresh complete DMEM containing appropriate concentrations of drugs was added into each well. A blank control well was set and each group was cultured and treated in triplicate. Cells were incubated at $37^{\circ} \mathrm{C}$ with $5 \% \mathrm{CO}_{2}$ for $48 \mathrm{~h}$, and the culture medium was discarded. The cells were gently washed twice with PBS for 2 min each time, and then $500 \mu \mathrm{l}$ methanol was added into each well for $3 \mathrm{~min}$. Any remaining solution was discarded, cells were washed twice with PBS, and Giemsa stain was added to completely cover the cells on the slides for $30 \mathrm{~min}$. Following staining, the cells were washed with pure water until the solution was colorless. Next, 1-2 drops of neutral gum (Bioworld Technology, Inc., St. Louis Park, MN, USA) were added onto a clean glass slide. A small corner of the coverslip was gently clamped using ophthalmic forceps, and the coverslip containing the cells was placed with the cells facing down. When neutral gum fully expanded along the coverslip, any remaining neutral gum was absorbed with absorbent paper. At the same time, the slides were laid flat to avoid the formation of air bubbles and were stored at room temperature until further use. The morphology of Giemsa-stained apoptotic cells was observed under the microscope and images were captured.

Cell cycle progression and apoptosis determined by flow cytometry. Cells were inoculated into 6 -well plates at a density of $1 \times 10^{5}$ cells per well and incubated overnight at $37^{\circ} \mathrm{C}$ with $5 \%$ $\mathrm{CO}_{2}$. Next, they were treated with $15 \mu \mathrm{M}$ CDV or $15 \mu \mathrm{M}$ DDP for $48 \mathrm{~h}$, digested with $0.25 \%$ trypsin solution (without EDTA), collected by centrifugation at a speed of $200 \mathrm{x} g$ for $2 \mathrm{~min}$ at $4^{\circ} \mathrm{C}$, and washed twice with cold PBS. Subsequently, $70 \%$ pre-cooled ethanol (diluted with PBS) was added to suspend the cells, and they were stored at $4^{\circ} \mathrm{C}$ overnight, or at $-20^{\circ} \mathrm{C}$ for a longer storage period. Cells were harvested by centrifugation at $200 \mathrm{x} g$ for $10 \mathrm{~min}$ at $4^{\circ} \mathrm{C}$, washed twice with pre-cooled PBS, centrifuged again and collected by discarding the supernatant. Pre-cooled PBS was added to suspend the cells and obtain a concentration of $1 \times 10^{6}$ cells $/ \mathrm{ml}$. Subsequently, the RNase A enzyme was added to a final concentration of $1 \mathrm{mg} / \mathrm{ml}$. The suspension were mixed and placed in a water bath at $37^{\circ} \mathrm{C}$ for $30 \mathrm{~min}$, followed by addition of propidium iodide (GT21008; Sigma-Aldrich) stain to a final concentration of $50 \mu \mathrm{g} / \mathrm{ml}$. Following gentle mixing, the cells were stored at $4^{\circ} \mathrm{C}$ to avoid light exposure and tested by flow cytometry. The red fluorescence at $490 \mathrm{~nm}$ was recorded using a microplate reader (Thermo Fisher Scientific Inc.), and the results were analyzed by CELLQUEST MODFIT LT computer systems (BD Biosciences, Franklin Lakes, NJ, USA) (9).

Expression levels of E6 and 533 proteins determined by western blot analysis. The effects of CDV and DDP on the expression levels of E6 and p53 proteins were detected by western blot analysis. Cytoplasm and nucleus extracts were prepared using a Nuclear and Cytoplasmic Protein Extraction kit according to the manufacturer's instructions. Total protein was extracted from HeLa cells, and a 15\% separation gel and 5\% stacking gel were used to separate the proteins. A total of $10 \mu \mathrm{l}$ of sample were loaded onto each well, the samples were run on the separation gel for $15 \mathrm{~min}$ and the stacking gel for $80 \mathrm{~min}$. Next, the gel was transferred to a membrane, which was then blocked for more than one hour. The following primary mouse antibodies were added to the membrane and incubated overnight at room temperature: Anti-human E6 (1:1,000), anti-p53 (1:1,000) and anti- $\beta$-actin (1:500). Following washing, the goat anti-mouse and anti-rabbit FITC secondary antibodies (both 1:5,000) were added and incubated for $1 \mathrm{~h}$ at room temperature. $\beta$-actin was used as a loading control. After washing with Tris-buffered saline with Tween 20 three times, the membrane was developed and an image was captured with Vilber Lourmat (Bio-Rad Laboratories Inc.).

Cell immunofluorescence staining. Sterile slides were inserted into 24-well plates. HeLa cells were plated into each well at a concentration of $5 \times 10^{4} / \mathrm{ml}$ and incubated at $37^{\circ} \mathrm{C}$ with $5 \% \mathrm{CO}_{2}$ for $48 \mathrm{~h}$. The culture media were discarded, and cells were washed twice with PBS for 1 min each time. Next, $4 \%$ cool paraformaldehyde was added for $10 \mathrm{~min}$ at room temperature. The cells were then washed three times with PBS for 1 min each time, and blocked with milk for 30-60 min at room temperature. Subsequently, the blocking solution was discarded, and mouse anti-human E6 and mouse anti-p53 antibody diluted in blocking solution were added. The plates were placed in an immunohistochemistry dampness box and then in an incubator at $37^{\circ} \mathrm{C}$ for $30 \mathrm{~min}$, or $4^{\circ} \mathrm{C}$ overnight. Cells were washed 2-3 times with PBS for $1 \mathrm{~min}$ each time and secondary fluorescent antibodies diluted by milk were added, placed in the wet boxes, followed by incubation at $37^{\circ} \mathrm{C}$ for $30 \mathrm{~min}$. The secondary antibody used was Alexa Fluor 594-conjugated donkey anti-rabbit IgG (1:400). Subsequently, the cells were washed 2-3 times with PBS for $1 \mathrm{~min}$ each time. Mounting solution containing 4',6-diamidino-2-phenylindole was dropped onto the glass slides. A tiny corner of the miniature coverslip was gently clamped by ophthalmic forceps and placed on the glass slide with the cells growing on the coverslip slide. Images of the cells were captured under fluorescence microscopes and analyzed by Image-Pro Plus 6.0 software.

Statistical analyses. Data were statistically processed and analyzed by SPSS version 17.0 (SPSS, Inc., Chicago, IL, USA). Measurement data are presented at a format of mean \pm standard deviation. Data were analyzed by one-way analysis of variance to compare the differences between groups, and $\mathrm{P}<0.05$ was considered to demonstrate statistically significant differences.

\section{Results}

Effect of CDV on HeLa cell viability, determined by MTT assay. As observed under an inverted microscope, the morphology of HeLa cells appeared to change significantly after treatment with CDV (Fig. 1A) and DDP (Fig. 1B). The treated cells with CDV and DDP had irregular shapes, smaller 


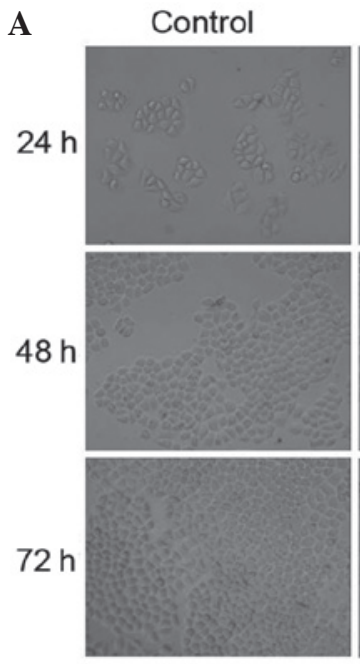

B

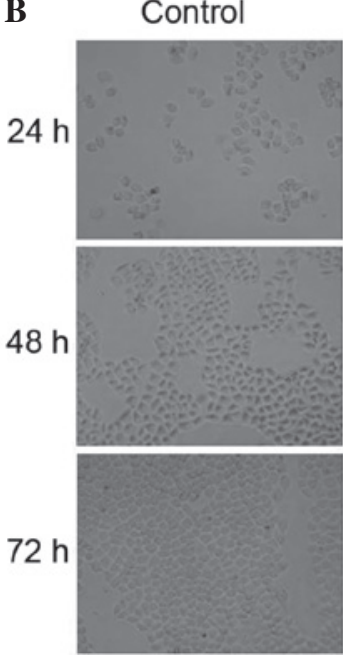

$2.5 \mu \mathrm{M}$

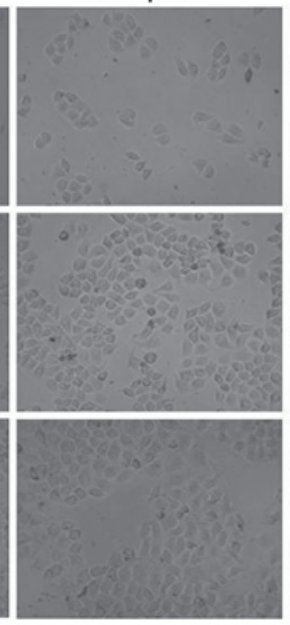

$2.5 \mu \mathrm{M}$

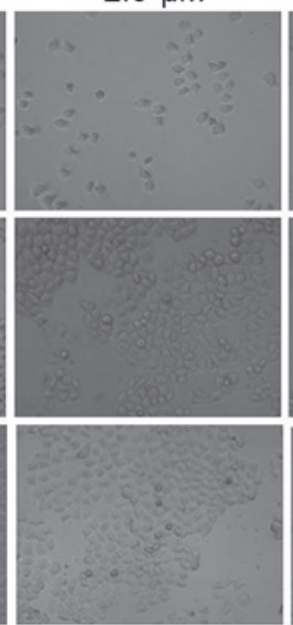

$5 \mu \mathrm{M}$

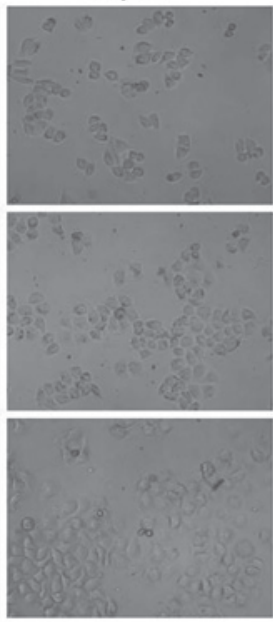

$5 \mu \mathrm{M}$
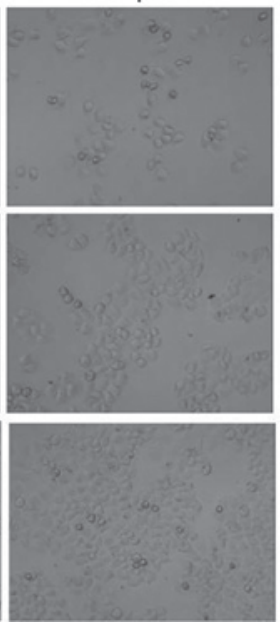

$10 \mu \mathrm{M}$

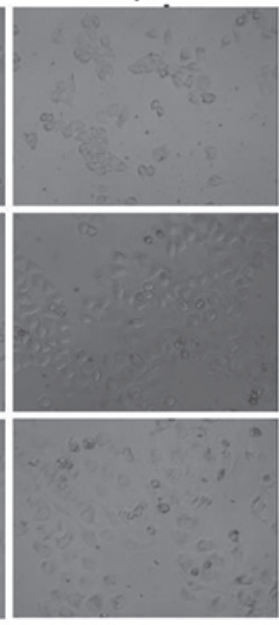

$10 \mu \mathrm{M}$

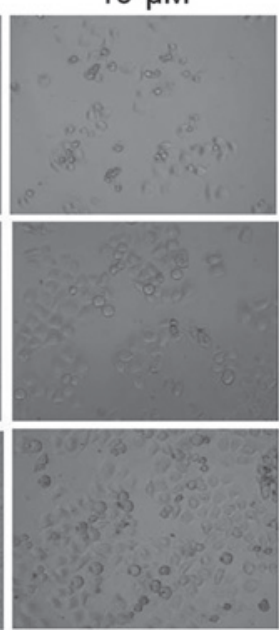

$20 \mu \mathrm{M}$

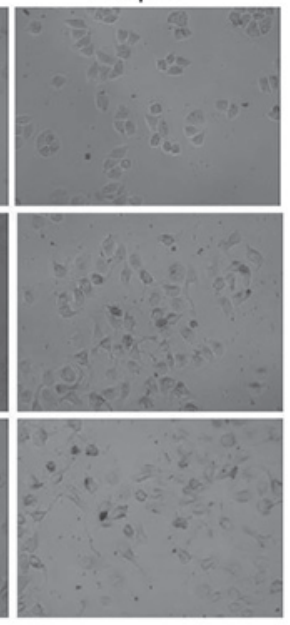

$20 \mu \mathrm{M}$

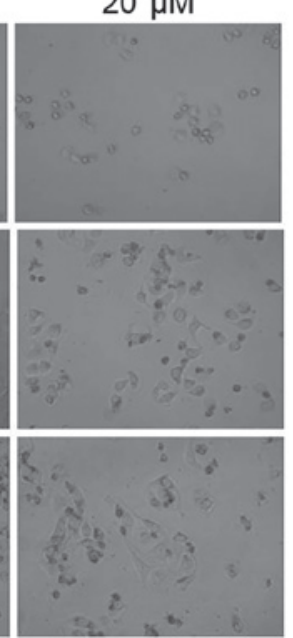

Figure 1. (A) Growth of HeLa cells treated with different concentrations of (A) cidofovir and (B) cisplatin, for 24, 48 and 72 h (magnification, x200).

sizes, dissociation of cell membrane, karyopyknosis and dark stained nuclei. HeLa cells in the control group were stained as light blue or light purple by Giemsa reagent, whereas apoptotic cells in the CDV and DDP groups were stained dark blue or dark purple. In the control group, cells were characterized by loose cytoplasm that were stained light purple, large nucleoli, and separated nucleoli and cytoplasm. However, the characteristics of cells in the CDV and DDP treatment group suggested that the size of the numerous cells was reduced, some cells were long and spindle-shaped, whereas agglutination and karyopyknosis in the nucleus chromatin were stained with a dark color, similar to the typical apoptotic cells. The results showed that treatment with CDV or DDP was able to induce apoptosis and morphological changes in HeLa cells. At the same time points, treatment with CDV and DDP had a similar inhibitory effect on the proliferation of HeLa cells in a concentration-dependent manner, with an increased number of apoptotic cells observed. Additionally, at the same drug concentrations, the two treatment groups presented increased numbers of apoptotic cells in a time-dependent manner. All these aforementioned observations demonstrated that CDV and DDP treatments can inhibit the proliferations of HeLa cells depending on the incubation time and drug concentration.
The inhibitory effects of CDV and DDP on the proliferation of HeLa cells changed markedly in time- and concentration-dependent manners, when compared with the proliferation observed in the control group (Fig. 2). Cell viability was reduced at higher concentrations and incubation times. Through this MTT cell viability detection and data analysis, it was concluded that the concentration of CDV and DDP that was able to inhibit the viability of HeLa cells by $50 \%$ after $48 \mathrm{~h}$ of treatment was $15 \mu \mathrm{M}$ for both drugs. Thus, the treatments of $15 \mu \mathrm{M} \mathrm{CDV}$ and $15 \mu \mathrm{M}$ DDP were adopted for $48 \mathrm{~h}$ in subsequent experiments in the present study.

Formation of HeLa cell colonies. As shown in Fig. 3, the results of Giemsa staining of HeLa cell colonies demonstrated that the CDV and DDP groups had a reduced number of colonies when compared with the control group. In addition, the cell survival rate of the CDV group (75.35 $\pm 1.14 \%)$ was significantly higher compared with that in the DDP group $(63.71 \pm 0.82 \%)$, demonstrating that cell proliferation was inhibited to a greater extent in the DDP group.

Apoptotic cell detection by Giemsa staining. As shown in Fig. 4, HeLa cells in the control group were stained as light blue or light purple by Giemsa reagent, while apoptotic cells 
A

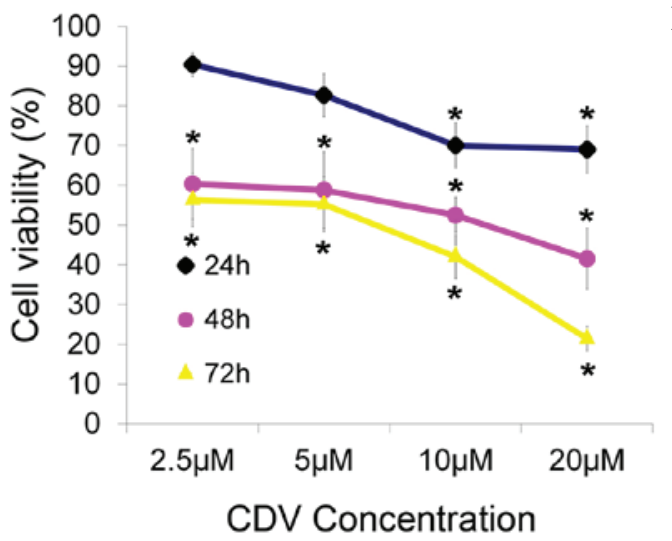

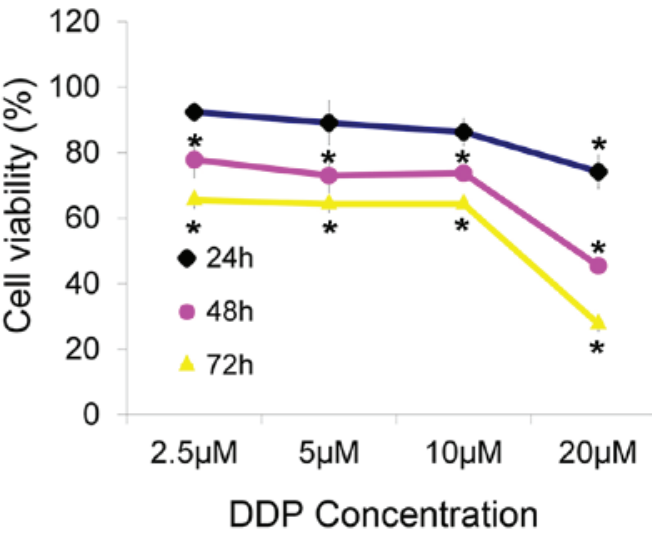

Figure 2. Cell viability of HeLa cells treated with (A) CDV and (B) DDP for 24, 48 or 72 h, as detected by MTT assay. Data were analyzed by one-way analysis of variance. Values are expressed as the mean \pm standard deviation in each group $(n=3)$. ${ }^{*}<0.05$ compared to the control group. CDV, cidofovir; DDP, cisplatin.

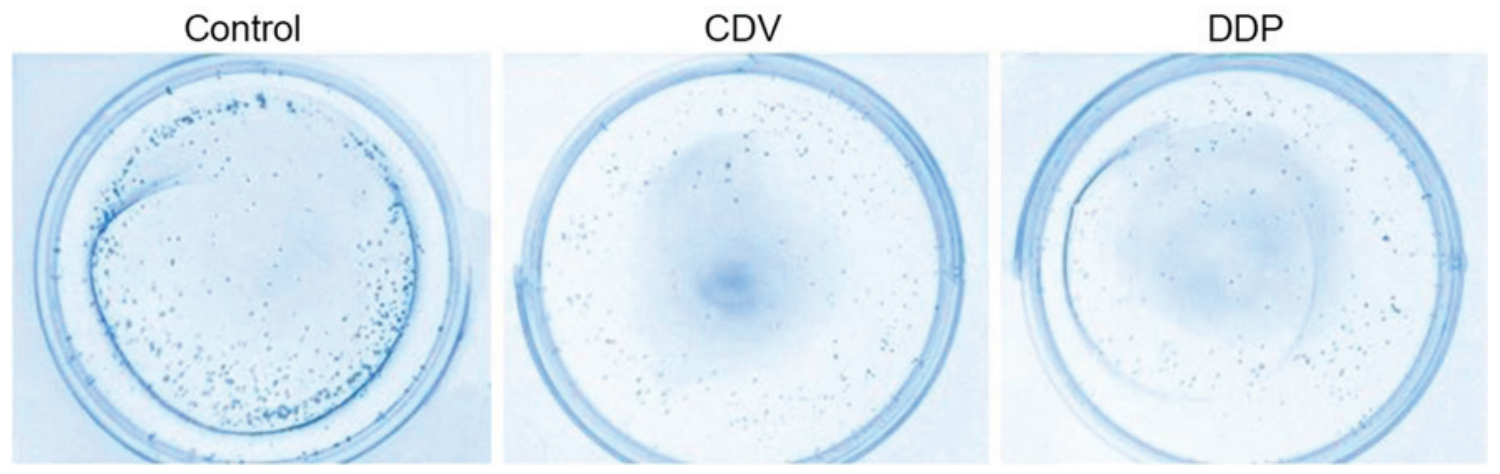

Figure 3. Colony formation of HeLa cells, observed using Giemsa staining. CDV, cidofovir; DDP, cisplatin.

in the CDV and DDP group were stained as dark blue or dark purple. In the control group, cells were characterized by loose cytoplasms that were stained light purple, large nucleoli, separated nucleoli and cytoplasms, and nucleoli that were visible with a large number of visible nucleoli. However, the characteristics of cells were observed by an inverted microscope (Figs. 4 and 5). The morphology of HeLa cells changed significantly. They exhibited irregular shapes, were smaller in size, had dissociation of the cell membrane, karyopyknosis and dark-stained nuclei. At the same time, the inhibitory function of CDV and DDP on the proliferation of HeLa cells increased as the concentration of the drugs increased. In addition, the proportion of HeLa cells increased as the concentration of drugs and the proportion of apoptotic cells increased, and at the same drug concentration the proportion of apoptotic cells increased with time. As shown in Fig. 5, with the arrows indicating apoptotic cells and apoptotic bodies. The size of apoptotic cells was reduced, the cytoplasm was condensed and the membrane was intact with a foaming phenomenon detected. At the late phase of apoptosis, nuclei were lysed into pieces, producing apoptotic bodies. Fig. 5 suggested that treatment with CDV or DDP was able to induce apoptosis and morphological changes in HeLa cells, and the effect of CDV was stronger. Therefore, these findings demonstrated that treatment with CDV or DDP was able to induce apoptosis and morphological changes in HeLa cells.
Cell cycle progression and apoptosis detection by flow cytometry. The results of cell cycle detection showed that CDV and DDP treatments had an impact on the progression of the HeLa cell cycle (Fig. 6A). Compared with the control group, the proportion of cells in $\mathrm{S}$-phase was significantly increased in the CDV group $(\mathrm{P}<0.05)$, demonstrating that $\mathrm{CDV}$ was able to block the HeLa cell cycle in the S-phase. In addition, the proportion of HeLa cells in G0/G1 phase and S-phase in the DDP group increased slightly, demonstrating that DDP possibly resulted in the arrest of certain HeLa cells in the G0/G1 phase and of others in the S-phase. These results indicated that the two drugs inhibited the progression of the HeLa cell cycle.

Furthermore, the results of the cell apoptosis test demonstrated that an apoptosis peak was evidently observed in the CDV and DDP groups (Sub-G0 peak), whereas this was not observed in the control group (Fig. 6B and C). This indicated that CDV and DDP induced the apoptosis of HeLa cells. The apoptosis rates of HeLa cells in CDV $(2.593 \pm 0.103 \%$; $\mathrm{P}<0.05)$ and DDP groups $(3.573 \pm 0.348 \%$; $\mathrm{P}<0.05)$ were significantly higher when compared with the rate in the control group.

Effects of $C D V$ and DDP on the expression levels of E6 and p53 protein. The results (Fig. 7; Table I) showed that, compared with the control group, the expression of E6 protein decreased subsequent to treatment with the two drugs. E6 expression in the CDV group decreased more significantly $(0.088 \pm 0.010$; $\mathrm{P}<0.01)$, compared with that in the DDP group $(0.100 \pm 0.017)$. 

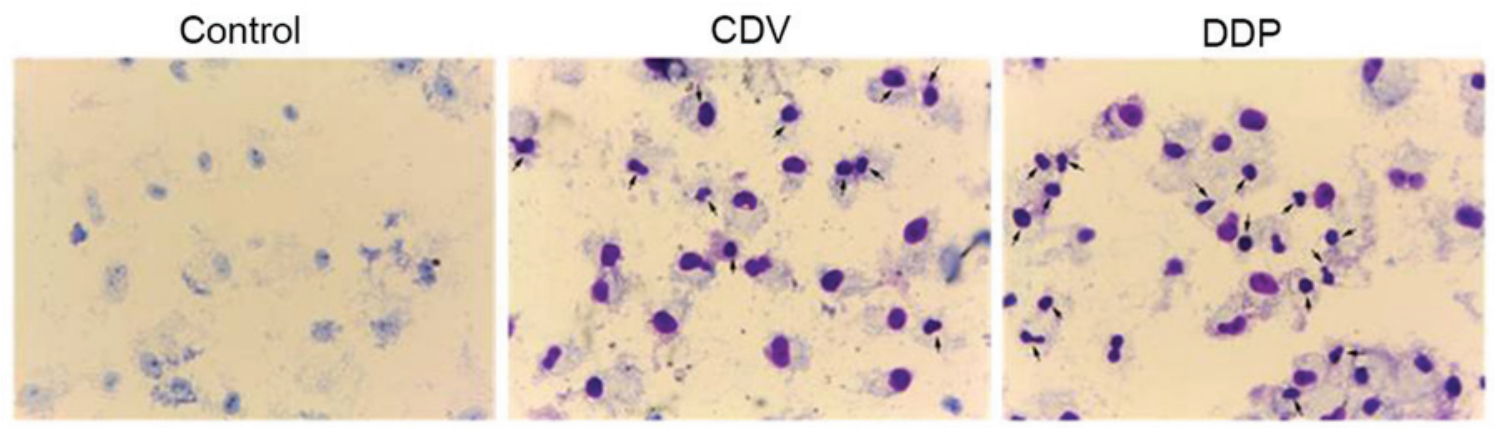

Figure 4. Detection of apoptosis of HeLa cells using Giemsa staining (magnification, x200). The arrows show apoptotic cell nuclei. CDV, cidofovir; DDP, cisplatin.
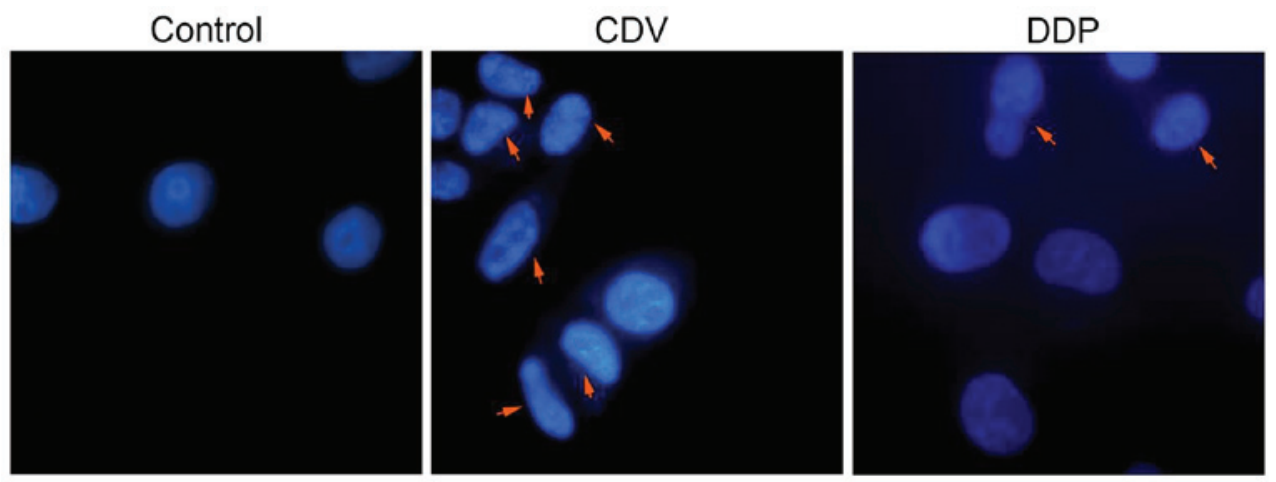

Figure 5. HeLa cells stained by DAPI (magnification, x1,000). The arrows show apoptotic cell nuclei. CDV, cidofovir; DDP, cisplatin.
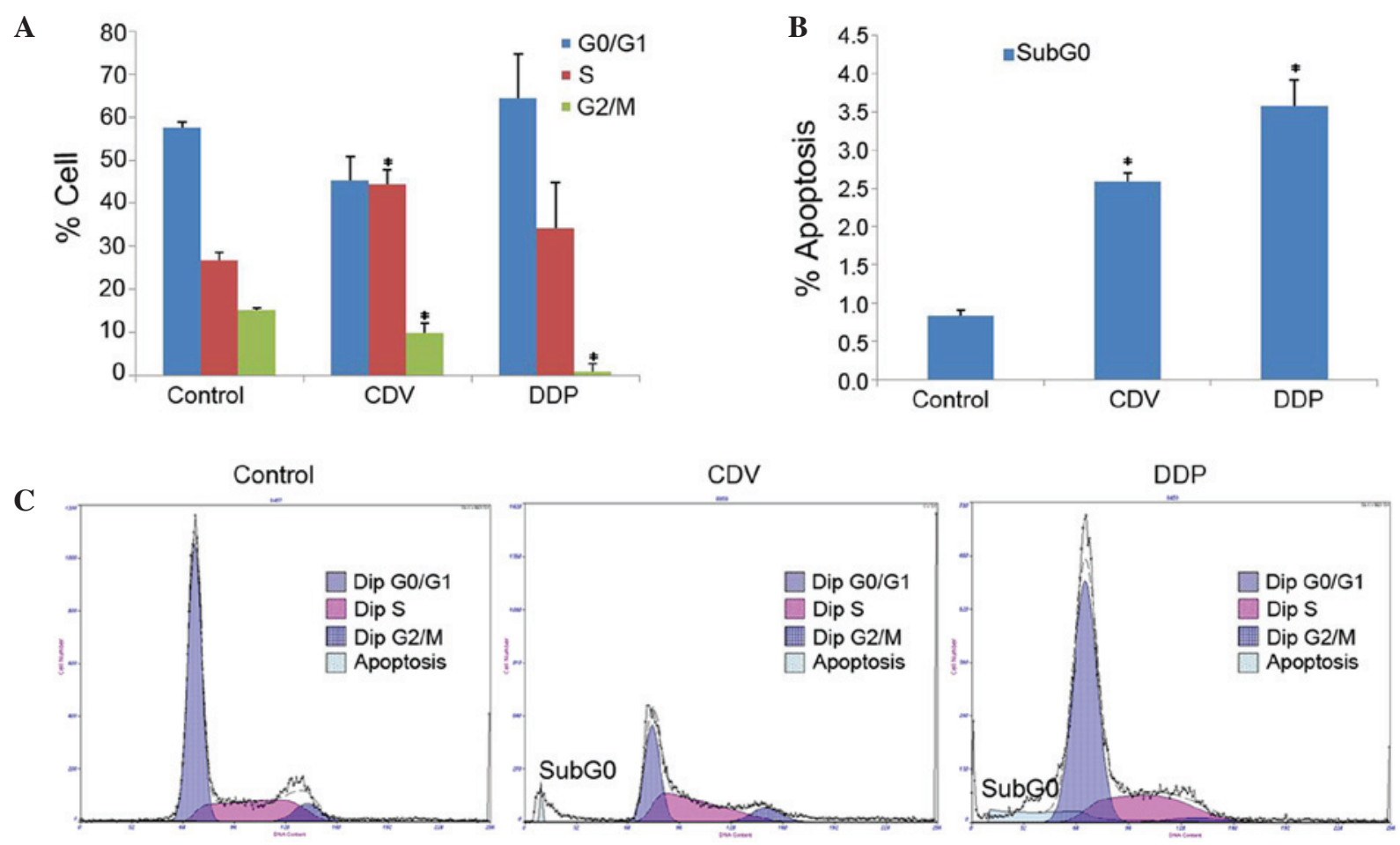

Figure 6. Cell cycle progression and apoptosis of HeLa cells. Histograms of (A) cell cycle distribution, and (B) apoptosis percentage. Values are expressed as the mean \pm standard deviation in each group $(\mathrm{n}=3)$. (C) Apoptosis of HeLa cells detected by flow cytometry. " $\mathrm{P}<0.05$ vs. the control group. CDV, cidofovir; DDP, cisplatin.

By contrast, compared with the control group, the expression of $\mathrm{p} 53$ protein increased subsequent to treatment with these drugs, with the expression increasing more significantly in the
CDV group $(0.103 \pm 0.012 ; \mathrm{P}<0.01)$. Overall, the decreases in E6 protein and the increases in $\mathrm{p} 53$ protein were not statistically significant. 
A

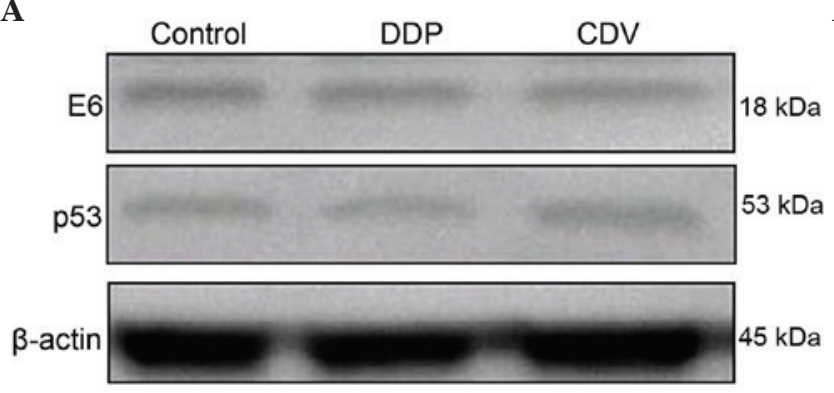

B

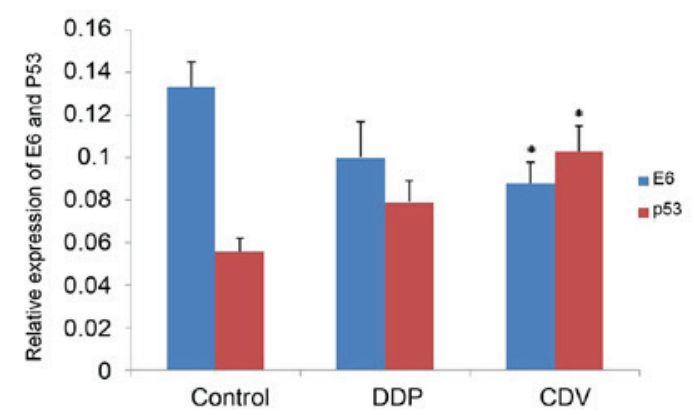

Figure 7. (A) Western blots and (B) quantified western blotting results, showing the expression levels of E6 and p53 proteins treated with CDV and DDP. Values are expressed as the mean \pm standard deviation for three wells in each group. ${ }^{*} \mathrm{P}<0.05$ vs. control group. CDV, cidofovir; DDP, cisplatin.
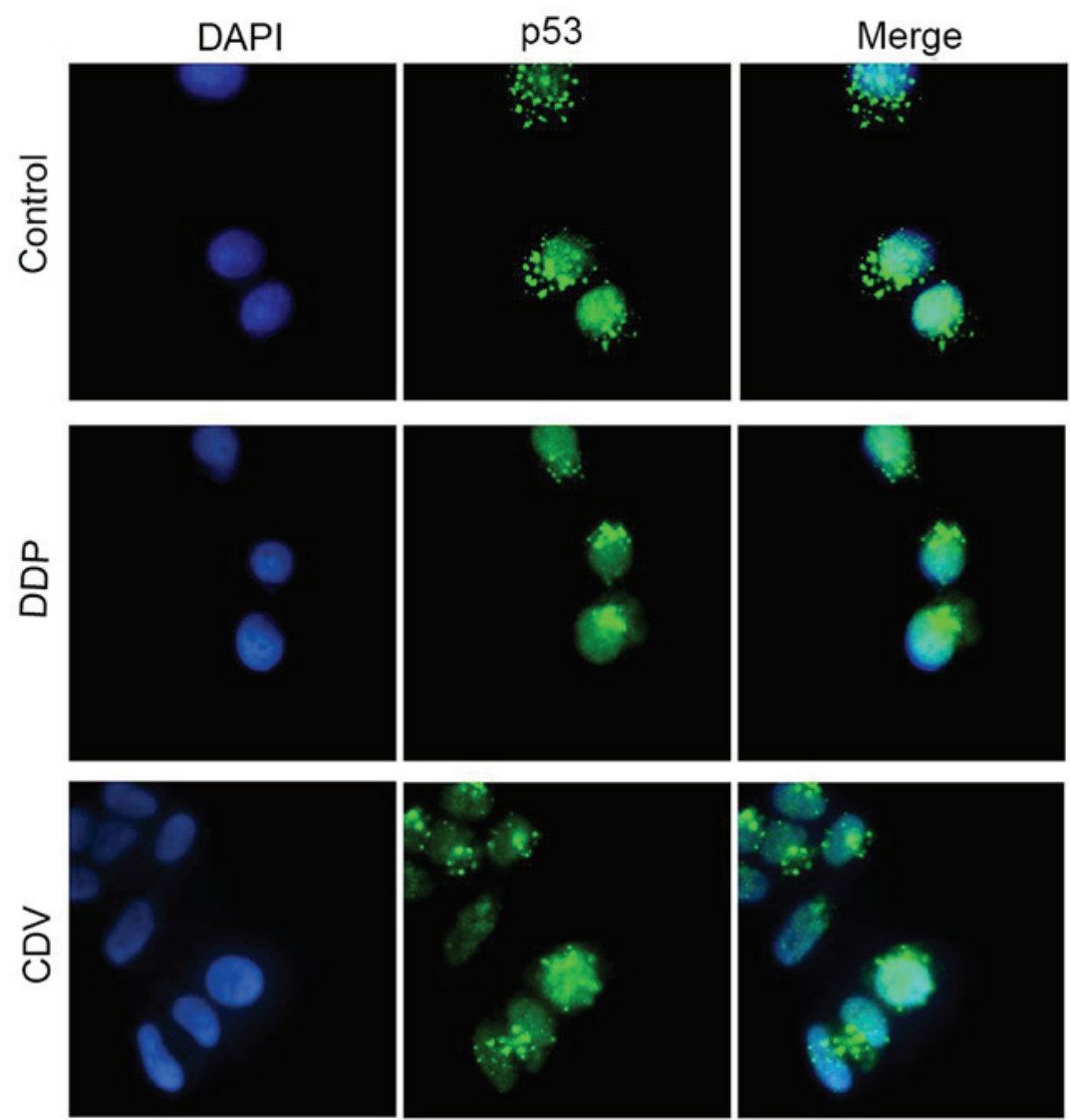

Figure 8. Immunofluorescence microscopy detection of p53 expression in HeLa cells treated by CDV and DDP (magnification, x1,000). Nuclei were shown in the DAPI-stained cell (blue) and p53 protein was shown as green fluorescence. CDV, cidofovir; DDP, cisplatin.

The results of immunofluorescence staining (Fig. 8) revealed that p53 mainly gathered in the nuclei of HeLa cells in the control group, while only a small part was distributed in the cytoplasm. However, compared with the control group, p53 protein accumulated mainly in the nuclei of cells treated with CDV or DDP, which was possibly caused by the decrease in E6 protein expression. This is assumed because analysis of other complex structures of the p53 core showed that the E6-binding surface of p53 was available for interaction not only in 'free' p53 tetramers but also in p53 molecules bound to DNA or other cellular proteins (10). These results were consistent with the findings of western blot analysis. In conclusion, cidofovir and cisplatin inhibited proliferation and induced apoptosis of HeLa cells. Furthermore, cidofovir may also be used for cervical cancer therapy.

\section{Discussion}

Cervical HPV vaccines can be divided into two types, including preventive and therapeutic vaccines. Preventive vaccines mainly include anti-HPV 16 and 18 bivalent vaccines, as well as anti-HPV 6, 11, 16 and 18 tetravalent vaccines, which can provide protection for nearly $70 \%$ of patients (11). 
Table I. Expression levels of E6 and p53 proteins treated with DDP and CDV.

\begin{tabular}{lcc}
\hline Groups & E6 & p53 \\
\hline Control & $0.133 \pm 0.012$ & $0.056 \pm 0.006$ \\
DDP & $0.100 \pm 0.017$ & $0.079 \pm 0.010$ \\
CDV & $0.088 \pm 0.010^{\mathrm{a}}$ & $0.103 \pm 0.012^{\mathrm{a}}$
\end{tabular}

Data were analyzed by one-way analysis of variance and are expressed as mean \pm standard deviation for three wells in each group. ${ }^{a} \mathrm{P}<0.05$ vs. control group. CDV, cidofovir; DDP, cisplatin.

DDP is widely used in anti-cancer treatment, since it is able to induce DNA damage, activate p53 and induce programmed cell death, thus making it one of the most common drugs in the treatment of cervical cancer (12). CDV was originally approved by the Food and Drug Administration for the treatment of acquired immune deficiency syndrome patients with retinitis caused by cytomegalovirus (13). In recent years, the clinical indications of CDV have been expanded. However, there are few studies focusing on using CDV as antiviral therapy (14).

In high-risk HPV infection, E6 targets $\mathrm{p} 53$ protein, binds to p53 through the E6-associated protein (E6-AP), induces permanent cell proliferation, and impairs the regulatory role of p53 in cell apoptosis and cell cycle arrest. Ultimately, all these effects of E6 led to cell immortalization and the occurrence of cervical cancer $(15,16)$. Studies have demonstrated that $\mathrm{p} 53$ protein serves an important role in cell cycle regulation and apoptosis, as well as in the progression and development of tumors (17). p53 is located in the nuclei, and can maintain cell genome stability by inhibiting cell proliferation, regulating the cell cycle and gene transcription, and activating programmed cell death (18).

The present study identified that CDV and DDP inhibited the growth and proliferation of HeLa cells. This inhibitory effect was depended on the drug concentration and incubation time, and was enhanced by higher concentration and longer treatment times. Under the same experimental conditions, DDP had a relatively higher inhibitory effect on the proliferation of HeLa cells compared with CDV. According to the results of flow cytometry, CDV and DDP had an impact on the HeLa cell cycle progression and induction of cell apoptosis. CDV caused HeLa cell arrest in S-phase, and DDP resulted in the arrest of certain HeLa cells in S-phase and others in G0/G1 phase. This indicates that the two drugs had inhibitory effects on the cell cycle progression of HeLa cells and can induce HeLa cell death; thus, CDV and DDP may restore the activity of p53 protein in certain ways.

Comparative analysis of the above findings suggests that CDV and DDP may reduce the expression levels of E6 protein or activate the p53 protein pathway in HeLa cells. The expression activity of p53 recovers under the effects of DDP, since DDP may reduce the E6 activity in HeLa cells directly or indirectly by binding with the sulfhydryl on the surface of E6. In addition, DDP and p53 protein competitively bind with E6 protein, which can cause the release of $\mathrm{p} 53$ and then recovery of p 53 expression, and ultimately inhibit cell proliferation and induce cell apoptosis (19). In the present study, western blot analysis revealed that, following treatment by CDV or DDP, the E6 protein levels in HeLa cells were reduced, resulting in an increase in p53 protein levels. This suggests that CDV and DDP are able to inhibit the expression of E6 protein in HeLa cells, and resume the activity of p53 protein. The study of Donne et al (20) showed that the upregulated expression of E6 mRNA was observed using semi-quantitative or quantitative polymerase chain reaction, which may be associated with the specific concentration of $\mathrm{CDV}$, the experimental time or the types of cells.

The expression of E6 protein has been shown to be associated with the location and translocation of $\mathrm{p} 53$ protein in the nuclei of HPV-positive cells (21). In cervical cancer cells, p53 tumor suppressor protein normally exists as wild-type. In HPV-infected cervical cancer cells, E6 combines with p53 protein into a stable E6-p53 complex by E6-AP. In HeLa cells without the presence of mouse double minute 2 homolog, HPV 18 E6 and E6-AP, which contain a nuclear export sequence, are capable of transporting the p53 protein to the cytoplasm (22). E6 can degrade p53 protein by the proteasome system in the nuclei and cytoplasm. These previous findings are consistent with the current study results showing that $\mathrm{p} 53$ protein can be observed in the nucleus and cytoplasm in the control group. Currently, it is considered that the degradation of p53 by HPV E6 is, at least partly, based on the nuclear export of the protein. The common mechanism involves E6-AP degrading and regulating p53 by shuttling between the nuclei and cytoplasm (23). In the current study, subsequent to CDV and DDP treatment, p53 protein in HeLa cells was mainly located in the nuclei rather than the cytoplasm. According to the results of western blot analysis, it can be inferred that CDV and DDP reduced the expression of E6 protein, activating the wild-type p53 protein expression.

In conclusion, treatment with CDV or DDP can inhibit the proliferation of HeLa cells in a concentration- and time-dependent manner. CDV and DDP can affect the expression levels of E6 and p53 proteins in HeLa cells, and consequently regulate cell cycle arrest and cell apoptosis. According to the findings of the present study, CDV can inhibit HeLa cell proliferation and induce cell apoptosis similar to DDP, which indicates that CDV may be a possible agent for cervical cancer treatment.

\section{Acknowledgements}

This study was supported by the Initial Project for Post-Graduates of Hubei University of Medicine (grant no 2015QDJZR08) and the Scientific Research and Technological Development Project of Shiyan Science and Technology Bureau (grant no. 16K70).

\section{References}

1. Drain PK, Holmes KK, Hughes JP and Koutsky LA: Determinants of cervical cancer rates in developing countries. Int J Cancer 100: 199-205, 2002.

2. Faridi R, Zahra A, Khan K and Idrees M: Oncogenic potential of Human Papillomavirus (HPV) and its relation with cervical cancer. Virol J 8: 269, 2011.

3. Ajay AK, Meena AS and Bhat MK: Human papillomavirus 18 E6 inhibits phosphorylation of p53 expressed in HeLa cells. Cell Biosci 2: 2, 2012.

4. Shi JF, Canfell K, Lew JB and Qiao YL: The burden of cervical cancer in China: Synthesis of the evidence. Int J Cancer 130: 641-652, 2012. 
5. Cox JT: Epidemiology and natural history of HPV. J Fam Pract Suppl: 3-9, 2006.

6. Tommasino M: The human papillomavirus family and its role in carcinogenesis. Semin Cancer Biol 26: 13-21, 2014.

7. Rosales R and Rosales C: Immune therapy for human papillomaviruses-related cancers. World J Clin Oncol 5: 1002-1019, 2014.

8. Collette DC and Zechel MA: Novel: treatment of atypical human papillomavirus-associated epithelial hyperplasia with cidofovir. J Oral Maxillofac Surg 69: 2383-2386, 2011.

9. Zhang YH, Peng HY, Xia GH, Wang MY and Han Y: Anticancer effect of two diterpenoid compounds isolated from Annona glabra Linn. Acta Pharmacol Sin 25: 937-942, 2004.

10. Travé $\mathrm{G}$ and Zanier K: HPV-mediated inactivation of tumor suppressor p53. Cell Cycle 15: 2231-2232, 2016.

11. Kjær SK, Frederiksen K, Munk C and Iftner T: Long-term absolute risk of cervical intraepithelial neoplasia grade 3 or worse following human papillomavirus infection: Role of persistence. J Natl Cancer Inst 102: 1478-1488, 2010.

12. Koivusalo R, Krausz E, Ruotsalainen P, Helenius $H$ and Hietanen S: Chemoradiation of cervical cancer cells: Targeting human papillomavirus E6 and p53 leads to either augmented or attenuated apoptosis depending on the platinum carrier ligand. Cancer Res 62: 7364-7371, 2002.

13. De Clercq E and Holý A: Acyclic nucleoside phosphonates: A key class of antiviral drugs. Nat Rev Drug Discov 4: 928-940, 2005.

14. Topalis D, Nogueira TC, De Schutter T, El Amri C Krecmerova M, Naesens L, Balzarini J, Andrei G and Snoeck R. Resistance to the nucleotide analogue cidofovir in $\mathrm{HPV}(+)$ cells: a multifactorial process involving UMP/CMP kinase 1. Oncotarget 7: 10386-10401, 2016.
15. Scheffner M, Werness BA, Huibregtse JM, Levine AJ and Howley PM: The E6 oncoprotein encoded by human papillomavirus types 16 and 18 promotes the degradation of $\mathrm{p} 53$. Cell 63: 1129-1136, 1990.

16. Werness BA, Levine AJ and Howley PM: Association of human papillomavirus types 16 and 18 E6 proteins with p53. Science 248: 76-79, 1990.

17. Jin Y, Wei Y, Xiong L, Yang Y and Wu JR: Differential regulation of survivin by $\mathrm{p} 53$ contributes to cell cycle dependent. Cell Research 15: 361-370, 2005.

18. Bargonetti J and Manfredi JJ: Multiple roles of the tumor suppressor p53. Curr Opin Oncol 14: 86-91, 2002.

19. Huang H, Huang SY, Chen TT, Chen JC, Chiou CL and Huang TM: Cisplatin restores p53 function and enhances the radiosensitivity in HPV16 E6 containing SiHa cells. J Cell Biochem 91: 756-765, 2004.

20. Donne AJ, Hampson L, He XT, Rothera MP, Homer JJ and Hampson IN: Cidofovir induces an increase in levels of low-risk and high-risk HPV E6. Head Neck 31: 893-901, 2009.

21. Abdulkarim B, Sabri S, Deutsch E, Chagraoui H, Maggiorella L, Thierry J, Eschwege F, Vainchenker W, Chouaïb S and Bourhis J: Antiviral agent Cidofovir restores p53 function and enhances the radiosensitivity in HPV-associated cancers. Oncogene 21: 2334-2346, 2002.

22. Stewart D, Ghosh A and Matlashewski G: Involvement of nuclear export in human papillomavirus type 18 E6-mediated ubiquitination and degradation of p53. J Virol 79: 8773-8783, 2005.

23. Hillemanns P, Jentschke M, Evans TG, Soergel P and Hass R: Detection of E6-AP as a potential therapeutic target in cervical specimen of HPV-infected women. Arch Gynecol Obstet 289: 1281-1286, 2014. 